\title{
I. Ueber die Lymphzellen.')
}

\section{Von Prof. Dr. A. Kossel in Berlin.}

M. H.! Wenn ich die Lymphzellen zun Gegenstand eines Vortrages gewählt habe, so geschah dies nicht allein wegen des grossen Interesses, welches die heutige Patlologie diesen Gebilden und den ihnell nahe verwandten Leukocyten des Blites entgegenbringt, sondern auch deshalb, weil die Chemie der Lymphzellen am besten geeignet ist, uns einen Einblick in die Chemie der Zelle iiberhaupt zll gewähren.

Ehe man es versucht, in das Wesen der chemischen Processe einzudringen, welche sich in der Zelle abspielen, miss man die Substanzen kennen lernen, welche bei diesen Reactionen in $\mathrm{Be}$ tracht kommen. Der chemischen Physiologie der Zelle muss eine chemische Beschreibung voraufgelien, demgemäss stehen unsere Kemntnisse ïber die Lyniphzellen, welche ja erst in den ersten Stadien ihrer Entwickelung begriffen sind, fast ausschliesslich auf einem descriptiven Standpunkt.

Die cliemische Beschreibung der Zelle hat sich nicht darauf zll beschränken, dass sie die Bestandtheile derselben alfzählt, ilıre Eigensehaften und ihre chemische Constitution klarlegt. Nicht minder wichtig ist es, die Vertheilung der Stoffe in der Zelle, die Beziehung der clemischen Bestandtheile zu den allatomischen Elementen zu kennen. Entsprechend diesen beiden Gesichtspunkten sind die Forschungen über die chemische Zusammensetzung der Lymphzellen zwei verschiedene Wege gegangell. Einerseits hat man es versucht, grössere Mengen voll Lymphzellen zu isoliren, um diese nach unseren chemischen Methoden zu verarbeiten, die Bestandtheile zll charakterisiren lund aus den Eigenschaften dieser Stoffe Reactionen für mikroskopische Erkennung abzuleiten. Andererseits ist man voll der anatomischen Betrachtung ausgegangen, man hat im mikroskopischen Bilde der Zelle mit Hülfe chemischer Reagentien gewisse Bestandtheile ansfindig zu machen versucht und es der makrocliemischen Forschung iiberlassen, die Natur dieser Stoffe festzustellen.

Eine notliwendige Bedingung fiil die Untersnchung in der erstgenannten Richtung ist die Beschaffung einer grösseren Menge von Lymphzellen. Man hat diese zum Theil aus den Lymphdrüsen, hauptsächlich aus der Thymusdrïse durcli Auspressen und Centrifugiren gewonnell. Die grosskernigen Zellen der Tlyymusdrüse erwiesen sich nach dieser Verarbeitnlly unter dem Mikroskop als wohlerhalten.

Welches sind num die Bestandtheile dieser Lymphzellen? Unter der grosseu Zahl der Stoffe, welche man in den Zellen ïberliaupt vorfindet, giebt es einzelne, die jeder entwickelungsfähigen Zelle angehören, die wir nie vermissen, wenn wir jugendliche Gewebe einer chemischen Untersuchung unterwerfen. Ich habe für diese den Namen primäre Zellstoffe vorgescllagen. Hoppe-Seyler hat sclion seit geraumer Zeit als solche Bestandtheile die Eiweisskörper, das Lecitlin, das Clolesterin und einige anorganische Stoffe unter diesen das saure phospliorsaure Kali, bezeichnet. Ich habe auch die Nucleïnstoffe als Angehörige dieser Reilie betrachtet. Alle diese Stoffe fanden sich aucl bei den Untersuchungen, welche Herr L. Lilienfeld in meinem Laboratorium angestellt lat, in den Lymplizellen vor. Ausser diesen priniären Stoffen waren aber aucl noch secundäre vorhanden, $d . h$. solche, die wir nicht in jeder entwickelungsfähigen Zelle vorfinden, die nur in gewissen Zellen und in gewissen Entwickelungsstadien auftreten. Solche sind das

1) Vortrag, gehalten im Verein für innere Medicin am 5. Februar 1894.
Glykogen, die Fette und das Protagon, Stoffe von der weitesten Verbreitıng, sodann ein Körper, der wegen seiner Mittelstellung zwisclien den Kohlehydraten und den aromatischen Substanzen von Interesse ist: der Inosit, und endlich eine Amidosälure, die man bisher nur vereinzelt in den Geweben vorgefunden hat: die Amidovaleriansäure, ein Zersetzungsproduct der Eiweisskörper.

Unter den genannten Substanzen llehmen nun die Nucleïnstoffe der Menge nach die erste Stelle ein. Es ist dies leicht verständlich, da der Kern fast den ganzen Raum der Zelle erfüllt. Die Nucleïnstoffe gehören bekanntlich $\mathrm{zu}$ einer Gruppe von Eiweisskörpern, welche man unter dem Namen der Proteïde zusammenfasst. Diese Proteïde sind Verbindungen der Eiweisskörper mit organischen odel anorganischen Stoffen, und zwar ist es in den Nucleïnen eine organische phosphorhaltige Gruppe, die „Nucleïnsäure“, welche an das Eiweiss angefügt ist.

Die chemische Untersuchung dieser Säure enthüllt uns organische Atomcoinplexe, welche von denen des Eiweisses durchaus verschieden sind. Ich habe mich seit mehreren Jaliren mit der Frage nach dem chemischen Bau der Nucleinnsäure beschäftigt und habe bei diesen Untersuchungen gefunden, dass in dem Molecül dieser Säure Atomgruppen vorhanden sind, welche Stickstoff- und Kohlenstoffatome in sehr enger Verbindung enthalten und die wir nach unseren heutigen chenischell Theorieen als ringförmige bezeichnell. Diese stickstofthaltigen cyclischen Gruppen, das Adenin, Gilanin, Hypoxanthin und Xanthin zeigen in ihrem chemischen Bau und iu ilıren Eigenschaften eine nahe Verwandtschaft zur Hainsäure. Meine Befunde brachten mich daher sofort anf den Gedanken, dass die Harnsäure aus den Nucleïnstoffen lervorgeht, und durch diese Untersuchung ist in der That das wiclitigste Argument gegebell, welches für dic Bildung der Harusäure aus den Bestandtheilen des Zellkerns angefuihrt werden kanı. Stadthagen und später Horbaczew ski haben diese Idee weiter verfolgt.

Ncben dell genannten, in ihrer Constitution der Harnsäure nahestehenden Stoffen gehen aber loch andere aus den Nucleîlısämrell hervor. In Gemeinscliaft mit Herrn Dr. A lbert Neumann konnte ich unter den Zersetzungsproducten noch eine schön krystallisirende Substanz von complicirter Zusammensetzung $\left(\mathrm{C}_{23} \mathrm{H}_{26} \mathrm{~N}_{8} \mathrm{O}_{6}\right)$ nachweisen; wir haben ihr den Namen 'Thymin beigelegt, da wir sie zuerst in der Nucleïnsäure der Thymuszellen aufgefunden habell. Mit diesen Stoffen ist aber die Zahl der organischen Verbindungen, die ihren Ursprung aus der Nucleïnsäure ableiten, noch lange nicht erschöpft. Das Molecül der Nucleïnsäure ist ein sehr complicirtes, es ist vielleicht ebenso mannigfaltig wie das der Eiweisskörper. Jedenfalls erhellt aus den von mir angeführten Tliatsaclien, dass neben dem Eiweiss noch eigenartige, vom Eiweiss durchaus verschiedene Atomgruppen in der lebenden Zelle thätig sind. Die Nucleïnsäure ist im Kern entwickelungsfähiger Zellen stets vorhanden, sie muss also für die Flinetionen dieses Organs unentbehrlich sein.

Sie ersehen aus diesen Ausfïhrungen, dass man zu völlig unrichtigen Vorstellungen gelangt, wenn man das Eiweiss als den wichtigsten Bestandtheil der lebenden Substanz, als den Träger des Lebens betrachtet. Ich nehme für die Nucleünsäure eine ebenso wichtige Rolle in Anspruch. Nichts berechtigt uns dazu, aus den chemischen Bestandtheilen der Zelle einen einzelnen herauszugreifen und in diesem das ganze Räthsel des Lebens zu suchen. 
Eine sehr merkwürdige Eigenthiimlichkeit der Nucleïnsäure ist ihre Fähigkeit, mit Eiweiss in Verbindung zu treten. Wie Altwa nn gezeigt hat, kann man eine solche Verbindung künstlich darstellen, wenn man Nucleönsäure in wässeriger Lösung zu einer Eiweisslösung hinzufügt. Es entsteht ein Niederschlag und dieser ist das Nucleïn. Mit der Bildung des Nucleïns aus der Nucleïnsäure und dem Eiweiss ist aber der Aufbau der Nucleïnstoffe noch nicht vollendet, denn wir finden in der Lymphzelle das Nuclein nocli wieder in Verbindung mit einem neuen Körper eiweissartiger Natur.

Ich möchte Ihre Aufmerksamkeit auf diese eigenthümliche Substanz lenken. Ich habe sie zuerst in den kernhaltigen rothen Blutkörperchen in Verbindung mit dem Nucleïn dieser Zellkerne vorgefunden und sie mit dem Namen Histon bezeichnet. Herr Lilienfeld hat sie später auch in den Lymphzellen der Thymusdrüse, sowie in allen zelligen Gebilden des thierischen Körpers nachgewiesen und eine Verbindung des Nucleïns mit Histon, das Nucleohiston, in reinem Zustand dargestellt.

Als ich die Eigenschaften des Histons zuerst kelnen lernte, glaubte ich in ihm einen der einfachsten Eiweisskörper, eine AIbumose, vor mir zu habell, aber meine neuen Untersuchungen haben mich zu einer anderen Ansicht gefülırt. Es ist mir gelungen, aus zwei Bestandtheilen thierischer Gewebe künstlich eine Substanz darzustellen, welche sich vom Histon nicht unterscheiden lässt. Der eine Component dieser Substanz ist in freiem Zustand bisher nur in den Spermatozoen des Laehses vorgefunden worden, es ist das von Miesclier entdeckte Protamin. Fiigt man eine Lösung des Protamins zu einer Lösung von Albumose (oder Eiweiss), so bleibt die Flüssigkeit zunächst klar, setzt man nun ein wenig Ammoniak hinzu, so entsteht ein Niederschlag, und dieser besitzt alle. Eigenthümlichkeiten des Histons. Wir liaben in dieser Entstehungsweise des Histons ein Gegenstïck zul der Entstehung des Nucleins. Das Protamin ist eine Base, es ist nur in alkalischer Lösung imstande, das Eiweiss zu binden, und es entsteht ein Proteïd von basischen Eigenschaften, das Histon; die Nucleïnsäure hingegen besitzt stark sauren Charakter und fällt das Eiweiss nur in saurer Lösung, auch das lierbei entstehende Proteîd, das Nucleïn, ist eine Säure.

Wenn das Histon wirklich aus Protamin und Albumose zllsammengesetzt ist, so müsste man es auch unter Bildung von Protamin zerlegen können. Ich habe mich bisher noch vergeblich bemüht, diesen Nachweis zu führen, ich finde aber diesen Misserfolg auch leicht verständlich. Das Protamin ist eine noch wenig bekannte Substanz, es ist durch keine besonders charakteristischen Merkmale gekennzeichnet, und seiner Auffindung unter den Zersetzungsproducten der Eiweisskörper stehen grosse Schwierigkeiten entgegen.

Dazu' kommt noch eine Eigenthiimlichkeit der Eiweisskörper, welche die Aufsuchung angefügter, sog. "prosthetischer" Gruppen unter Umständen ganz ausserordentlich erschweren kann. Das Eiweiss ist imstande, die anfangs nur locker angefügte Gruppe allmählich immer fester und fester zu binden und sie - sit venia verbo - in das Innere des Molecüls aufzunehmen. So ist es zu erklären, dass wir Verbindungen von Eiweiss - und Nucleïnsäure vorfinden, die ganz locker sind, und andere, aus denen man die angefügte Nucleïnsäure nicht wieder frei machen kann, und so werden die Schwierigkeiten der Loslösung angefügter Stoffe vom Eiweissmolecül verständlich.

Am besten wird diese merkwürdige Eigenschaft der Eiweisskörper durch die von Schmiedeberg untersuchten Verbindungen von Eiweiss mit den Oxyden des Eisens illustrirt; hier zeigt sich deutlich, wie das Eiweissmolecül auch künstlich zI einer mehr oder weniger innigen Aufnahme des Eisens veranlasst werden kann.

Diese Loslösing der angefügten Gruppen, welche den Manipulationen des Chemikers so schwer gelingt, wird von deli in der Zelle vorhandenen Kräften anscheinend mit grosser Leichtigkeit vollzogen. In den unreifen Spermatozeen des Lachses findet sicl Histon, beim Reifungsprocess wird aus diesem das Protamin in Freiheit gesetzt, es ist in den reifen Organen in beträchtlicher Menge vorhanden und leicht nachzuweisen. Im ruhenden Zellkern ist die Nucleïsäure an Eiweiss gebunden, bei der Mitose wird sie losgelöst und erscheint als ungepaarte Säure.

Man könnte die Frage aufwerfen, wie es denn möglich ist, diesen Process unter dem Mikroskop zu verfolgen. Um dies verständlich zu machen, muss ich an die Untersuchungen ton $\mathrm{Ehr}$ lich erinnern, welcher die organischen Farbstoffe zuerst in rationeller Weise anwandte, um die chemischen Eigenthümlichkeiten gewisser Zellstoffe zu charakterisiren. Wenn man eine Zelle mit dem Gemiscl einer Farbbase und eines sauren Farbstoffs behandelt, so nehmen gewisse Theile die Farbbase, z. B. das Methylgrün, andere die Farbsäure, z. B. das Säurefuchsin auf. Herr Lilienfeld und gleichzeitig Herr Posner haben das Verhalten der Ehrlichschen Mischung von Methylgrün und Säurefuchsin zu den Eiweiss- körperı und zu einem von mir dargestellten Präparat von Nuclë̈nsäure untersucht, und beide sind zu dem Resultat gekommen, dass die Eiweisskörper den sauren, die Nucleïnsäure den basischen Farbstoff aufnelimell. Aus den Versuchen des Herrn Lilienfeld iiber das Verhalten. der Nuclë̈nstoffe ergiebt sich, dass die Nuclë̈nsäure eine rein grüne, das Histon eine rein rothe Färbung annimmt. In dell aus Eiweiss und Nuclë̈nsäure zusammengesetzten Verbindungen ist der Farbenton ein gemischter, und zwar ist er in dem ziemlieh stark sauren Nucleïl ein blaugrüner, in dem neutralen Nucleohiston ein violetter.

Man ist also durch die Farbenanalyse in den Stand gesetzt, die reine Nucleinsäure mikı'oskopisch nachzuweisen, und es zeigt sich bekanntlich, dass in den rulienden Kernen der Lymphzellen der violette Ton vorherrscht, während bei der Mitose und in allderen plysiologischen Zuständel die der ungepaarten Nucleïnsäure entsprechende grüne Färbung durch das Ehrlich'sche Gemisch zum Vorschein gebracht wird.

Die Bedingungen, unter welchen das Freiwerdell der Nuclënsäure in den Zellen stattfindet, und die Verhältnisse der mehr oder minder festen Bindung all das Eiweiss ${ }^{1}$ ) sind bisher noch wenig erforscht. Durch die gemeinsam mit Herril Dr. Albert Neumann angestellten Versuche konnte ich feststellen, dass die Lyinphzellen der Thymusdrüse von allen bisher untersuchten Organen zur Darstellung der freien Nucleïnsäure am besten geeignet sind. Aus 1 Kilo gereinigten Tllymusgewebes erhieltell wir nach einem wenig eingreifenden Verfahren $25 \mathrm{~g}$ Nucleinnsäure, aus 1 Kilo Milz 5,4 g, aus Pankreasgewebe gelang die Darstellung der freien Säure nach der gleichen Methode nicht, obgleich in diesem Organ die Verbindung der Nucleïnsäure mit Eiweiss in sehr erheblichen Quantitäten vorhanden ist. Die Nucleïnsäure ist also in den verscliedenen Organen mit verschiedener Festigkeit gebunden.

Eine Vorstellung von der Function der Nuclernsäure in der lebenden Zelle können wir erst dann erhalten, wenn wir eine tiefere Einsicht in die clemiscle Constitution derselben gewonnen haben, aber wir haben in der eiweissbindenden Fähigkeit der Nucleïnsäure doch schon eine Eigenschaft derselben kennen gelernt, welche bei der Beurtheilung ihrer physiologischen Rolle in Erwägung gezogen werden muss. Schon vor längerer Zeit machte icl die Beobachtung, dass organisirte Theile, welehe man in eine Lösung der Nucleïnsäure bringt, schwell opak werden und absterben, indem das Eiweiss sofort mit der Nucleïnsäure in Verbindung tritt. Ich machte schon damals darauf aufmerksam, dass auf diese Weise ein Verständniss für die bactericide Wirkung gewisser Zellen gegeben werden kann.

Mein Bruder, Herr Dr. H. Kossel, hat die Einwirkung der Nucleïnsäure auf die Mikroorganismen zum Gegenstand einer eingehenden Untersuchung gemacht und ist dabei zu dem Resultat gekommen, dass die Nucleïnsäure selbst in einer Verdünnung, von $0,5 \%$ noch eine abtödtende Wirkung auf Cholerabacillen, Typhus: bacillen, Streptococcen, Staphylococcen ausübt. Die Zeit, in welcher sich diese Wirkung offenbart, ist je nach der untersuchten Species eine verschiedene. Die Cholerabacillen erfordern nur eine Einwirkung von wenigen Minuten, während Streptococcen und Staphylecoccen bei dieser Concentration erst nach mehreren Stunden abgestorben sind. Zum Vergleich wurde die Wirkung einer Essigsäure von gleicher Acidität studirt. Hier erfolgte die abtödtende Wirkung - wenn sie iuberhaupt eintrat - erst nach längel'er Zeit. Die Gegenwart von Eiweiss verlangsamt die bactericide Wirkung der Nuclernsäure, hebt sie aber nicht auf.

Bekanntlich hat man sich schon seit längerer Zeit bemïht, die bacterientödtende Wirkung der Zellen auf gewisse von der Zelle producirte Stoffe zuruickzufïhren. In der Nucleïnsäure ist ein chemisch gut charakterisirter Stoff bekannt geworden, welcher diese Wirkung selbst bei Gegenwart von Eiweiss ausüben kann. Die Zelle besitzt in ihr eine Waffe gegen die Mikroorganismen. Es ist bisher zwar noch keine Beobachtung vorhanden, welche uns beweist, dass diese Waffe..wirklich angewandt wird, aber wirt dïrfen wohl kaum annehmen, dass sie nur zum Sclimuck diene. Es ist sehr bemerkenswerth, dass die Lymphzellen von allen untersuchten Organen diejenigen sind, aus denen die Nucleynsäure am leichtesten. ohne tiefer greifende chemische Einwirkungen dargestellt werden kann.

Diese Betrachtungen sind der Lehre Metschnikoff's von der Phagocytose leicht anzupassen. Ich zweifle nicht daran, dass die Nucleinsäure bei diesen bactericiden Processen eille Rolle spielt; aber ebensowenig, wie man die Phagocytose als den einzigen Apparat zum Schutz des Organismus gegen die organisirten Krankheitserreger betrachten darf, ebensowenig ist auch die Nucleïnsäure der einzige Stoff, dem diese Wirkung in den Zellen zukommt. "Die von

1) Wahrseheinlich ist auch das quantitative Verhältniss żwischen $\mathrm{Nu}$. eleinsäure und Eiweiss von Einfluss auf die Festigkeit der Bindung. 
Hankin, Bitter, Christmas, Vaughan u. a. untersuchten bactericiden Stoffe sind nicht ohne weiteres auf die Nucleïnsäure zu beziehen.

M. H.! Ich glaube, durch diese Darlegungen erwiesen zu haben, dass die chemischen Bestrebungen auf dem Gebiete der Zellenlehre mit den wichtigsten Problemen der heutigen Medicin Berührung finden. Bisher freilich lraben sich unsere Kenntnisse über die Pathologie der Zelle fast ausschliesslich auf anatomischer Grundlage aufgebaut. Man hat mit Hülfe des Mikroskops die gesetzmässige Aufeinanderfolge der Erscheinungen studirt und durch rein morphologische Betrachtung gezeigt, wie im Gebiete der krankhaften Vorgänge eine Gestaltung mit Nothwendigkeit auf die andere folgt, wie die Zelle ihre Forn verändert und ihren Ort wechselt.

Durch solche Untersuchungen können wir zwar ein zusammenhängendes Bild von dem Ablauf der Gestaltungsvorgänge erhalten, aber wir gewinnen kein Verständniss für den ursächlichen $\mathrm{Zu}$ sammenhang der Erscheinungen. Dies Verständniss, das eigentliche Ziel aller physiologischen und pathologischen Studien, ist nur mit Hülfe chemischer Methoden und chemischer Betrachtungsweisen zu erreichen. Niemand zweifelt daran, dass die Vorgänge der Ernährung und des Wachsthums, welche alle Thätigkeiten der Zelle beherrschen, auf chemischen Processen beruhen und dass die. lebendige Kraft, welche bei der Reizung frei wird, aus chemischen Umsetzungen hervorgeht.

Die chemische Untersuchung der thierischen Gewebe, von deren Ergebnissen naturgemäss der weitere Ausbau der Zellenlehre abhängt, hat in neuerer Zeit noch einen besonderen Antrieb erhalten. Die ätiologischen Forschungen über die Infectionskrankheiten haben uns ausser der Kenntniss der Bacteriengifte auch eine Vorstellung von denjenigen Mitteln verschafft, durch welche der thierische Organismus sich gegen die eindringenden Krankheitserreger schützt. Diese Mittel beruhen auf der Bildung chemischer Producte, welche theils von den eingedrungenen Mikroorganismen, theils von dem erkrankten Körper selbst geliefert werden. Selbstverständlich muss unser Streben dahin gerichtet sein, eine Einsicht in die Natur dieser schützenden Stoffe zu gewinnen, um sie in rationeller Weise therapeutisch zu verwenden.

Diesen Bestrebungen gesellen sich andere hinzu, welche ebenfalls zu den natürlichen Hülfsmitteln des Thierlörpers greifen. Man hat die Erfahrung gemacht, dass in einzelnen Organen, zum Beispiel in der Thyreoidea, Stoffe enthalten sind, welche dem Körper einen Schutz gegen gewisse Krankheiten gewähren können, also auch hier heisst es: chemische Untersuchungen, chemische Methoden!

Durch die heutige Entwickelung der Medicin sind der Thierchemie neue Aufgaben von unabsehbarer Tragweite erwachsen. Freilich entsprechen die Hülfsmittel, welche der physiologischen Chemie heute zu Gebote stehen, diesen grossen und schwierigen Problemen durchaus nicht. Der Entwickelung ihrer eigenartigen Methoden und der Ausbildung genügender Arbeitskräfte sind grosse Schwierigkeiten bereitet worden. Man hat ihre Erhebung zu einer selbstständigen Wissenschaft zu hindern gesucht, und vielfach hat man sie auf die Hinterstuben fremder Institute verwiesen. Die Folgen dieses Systems machen sich jetzt zum Nachtheil der gesammten Medicin geltend. Heute erwartet nicht nur die theoretische Wissenschaft, sondern auch die praktische Heilkunde von der Thierchemie die Lösung der wichtigsten Probleme. Aber die Schwierigkeiten, welche der erfolgreichen Bearbeitung dieser Aufgaben entgegenstehen, sind lediglich äussere, lassen Sie uns hoffen, dass dieselben bald überwunden sein werden! 